\title{
Process Information Modelling (PIM) Concept for On-site Construction Management: Hong Kong Case
}

\author{
Wen Pan ${ }^{1 *}$, Bahriye Ilhan², Thomas Bock \\ ${ }^{1}$ Chair of Building Realisation and Robotics, Technical University of Munich, Arcisstrasse 21, 80333 Munich, Germany \\ 2 Department of Architecture, Faculty of Architecture, Istanbul Technical University, Taskisla Campus, 34367 Sisli / Istanbul, Turkey \\ * Corresponding author, e-mail: wen.pan@br2.ar.tum.de
}

Received: 15 June 2018, Accepted: 27 August 2018, Published online: 29 November 2018

\begin{abstract}
When compared to the manufacturing industry, the advances of intelligent and seamless project management approaches lag behind in the construction industry. This is due to the complexity of the construction projects as well as various stakeholders who acquire specific interest of the project. For instance, public building construction has many sub-tasks and numerous systematised working processes such as planning, mobilisation, scheduling, procurement and controlling. The management of the processes of each phase of the project life cycle is of great importance in order to prevent project delay and cost overrun. A particular importance is required if construction robotics is involved since precise process and scheduling information as well as feedback are critical to ensure each task is completed accordingly. This paper proposes a holistic system approach, which focuses on the process-oriented management for on-site construction. To succeed, the Process Information Modelling (PIM) concept is introduced. The main goal of PIM is to integrate Building Information Modelling (BIM) for extensible solutions with a process-based database platform, which allows for smooth data transfer and supports continuous data sharing among all stakeholders. The overall aim is to develop a collaborative way of planning, designing, producing, assembling and managing the entire project life cycle strategy. Digital documentation, simulation and realtime data are produced progressively to facilitate decision-making. A case study applying the PIM concept for the façade painting task by a painting robot for an on-going consultancy project commissioned by the construction industry council (CIC) in Hong Kong is presented. This paper is the revised version of the paper that has been published in the proceedings of the Creative Construction Conference 2018 (Pan et al., 2018).
\end{abstract}

\section{Keywords}

construction automation, Hong Kong, on-site construction management, process information modelling, PIM

\section{Introduction}

Many industries have entered the "Industry 4.0" paradigm, which offers smart manufacturing, smart realtime Data Acquisition, Internet of Things (IoT), Cloud Computing, Cyber-Physical System (CPSs), and Big Data Analytics (BDA). This trend has increased productivities and enhanced competitiveness of those industries that have adopted an advanced integration between the physical world and the digital one (Elkaseer et al., 2018). For many years, many researchers have agreed that efficient integration and coordination of design, construction, and management data can potentially benefit the overall performance of the construction industry (Higgin and Jessop, 2013). Recently, other industries are submerged with data; the construction industry is not an exception to this unprecedented trend. In each step of a construction project, the project team will be dealing with enormous datasets, which amongst various professions and the decisions that have been made, could impose serious implications on the construction project. Currently, BIM technologies are being considered in dealing with multi-dimensional CAD information systematically, and in improving data integration among cross-disciplinary collaboration across the industry and between the key stakeholders.

Even though BIM can cover the construction project span from early stages to the completion of the project, the implementation of BIM technologies is often fragmented in most of the construction projects. This is partly due to the fact that in practice BIM does not effectively categorise or integrate the most relevant data and distribute the information to the most desired stakeholder. A piece of information 
cannot function alone without specific protocols and relationships behind it (Dossick and Neff, 2010). The BIM technologies should be able to provide a platform where the data collected from each phase of the project that can be integrated as well as interoperating between various applications (Eastman et al., 2013). Therefore, the concept of the next generation of BIM is not merely just gathering information or the use of several technical tools, but managing information across diverse collaboration and interrelationship of the key stakeholders. Additionally, it is essential to deliver the right information to the right place for the right people at the right time (Jernigan, 2008). Process Information Modelling (PIM) has the potential to be developed as the next generation BIM, which will enhance information integration, yet focus on the process of each construction tasks and the relationship between each attribute. In this case, the attributes can be people, products, processes or technologies. By doing so, it helps the project team identify challenges of execution from both a technological and social approach, and proactively provides feasible solutions (Trist and Bamforth, 1951). The detailed description about PIM is demonstrated in the later section.

This paper proposes a scenario in conjunction with the ongoing consultancy project commissioned by the Construction Industry Council (CIC) in Hong Kong. It hypothesises the PIM concept will be imperatively applied to the utilization of a façade painting robot. In general, the Hong Kong Public Housing Construction (PHC) sector faces three major challenges: (1) to satisfy the increasing demand; (2) to achieve an affordable price and (3) to address demographic changes. Accordingly, the CIC commissioned the Chair of Building Realization and Robotics ( $\left.\mathrm{br}^{2}\right)$ at Technical University of Munich (TUM) to research and develop construction robots and automation strategies that are tailor-made for the PHC in Hong Kong. The proposed external painting robot provides an opportunity to develop and validate the PIM concept. The implementation of construction robotics will trigger a series of changes in the construction sequence and potentially revolutionise the construction industry as a whole. It is the commitment to meet the challenges of the collaboration of the academia and key stakeholders to launch a significant attempt for developing a tailor-made process-oriented approach with respect to the current BIM technologies. Although, research has been done based on the topics of BIM, still limited research topics were conducted related to the topic of how to implement BIM technologies when adopting robotic and automated technologies in the construction project. Few questions arise from the research. For example, when implementing robotic and automation, the operational methods and the working process will dramatically differ from the conventional way, so are the existing BIM technologies still adequate to handle the tasks? If not, is the proposed PIM concept able to tackle the challenges and how? This research forms the backbone for developing PIM applications in the future. However, due to the lack of available resources and complexity of the construction process, the PIM applications can only be conceptualised.

Consequently, the authors evaluate the current BIM and big data technologies through an extensive literature study to explore the potential constraints within each key project phases and exam how to transfer those constraints into opportunities for the construction sector and beyond. The proposed PIM concept offers a practical approach, which can be used as a guideline to integrate and distribute information and enhance decision-making procedures during the design, precedent and tendering, logistics, construction and facility management phases. As a result, the proposed PIM approach can yield a huge change in how the construction industry handles such large volumes of heterogeneous data as well as enhance information acquisition and integration, which both provides real-time data sharing among all key stakeholders. In addition, it lays a foundation for developing a practical PIM application in the future.

\section{Building Information Modelling (BIM) and Process Information Modelling (PIM) 2.1 BIM}

Recently, BIM is one of the most promising developments in the Architecture, Engineering and Construction (AEC) industries. BIM became more influential within construction projects, which commonly are used for the design, visualization, planning, facilities management and cost estimating purposes. Using modern modelling tools, such as Revit Architecture, ArchiCAD or Tekla Structures, the content produced by architects, designers and engineers have evolved from traditional 2D-drawings, sketches and written specifications to parametric, object-oriented 3D-models embedded with information to describe any building or facility in detail (Pan et al., 2017). With BIM technology, an accurate virtual model of a building is digitally constructed (Azhar et al., 2008).

When integrated efficiently, the computer-generated model contains precise geometry and relevant data necessary to support the construction, fabrication and procurement activities involved in the project (Eastman et 
al., 2013). Although BIM applications claim the seamless integration of data from each project phase, in practice it is less evident how accurate and rapid the data has been passed on and how the data has been utilised. This may result from the availability, compatibility and interoperability between raw data and the applications. These aspects determine if the data can be transferred, integrated and responded in real-time. On the other hand, BIM application is a knowledge-based and object-oriented approach that aims to digitally and visually represent the real world situations, in other words, it can be considered as the identical twin of the real world. This might sound unrivalled. However, when implementing construction robots or carrying out a complex construction project, understanding only the real world condition is not enough. A know-how based, interactive, proactive and responsive extension of BIM is required (Harty et al., 2010).

\subsection{PIM}

The widespread use of digital technologies will lead to huge amounts of data being generated throughout the construction process. Some of the data is well understood by the stakeholders, for instance, Computer-aided Design (CAD) data, Excel data, and 3D virtualisations. On the other hand, some of the other data may be less familiar to the stakeholders, and it greatly depends on their experience, background and professional field. The main objective of PIM is to make sure that everyone understands the data correctly; to predict what is going to happen in the future based on the existing information variables. Specific actions need to be taken to analyse the risks and challenges that might occur and to recommend the options to the decision maker in real time (Calie, 2017).

PIM application is a process-oriented, case-focused approach that provides detailed information about a specific task. It then breaks down into smaller, manageable data and is distributed to the right stakeholder at the right time. The recipient can plan and react to the distributed data by following a guideline generated by PIM. The main feature of PIM is to optimize the entire construction process - rather than optimizing some parts and neglecting the others - by offering rapid, consistent data management and providing interactive, proactive, responsive data integration. In addition, it is equipped with cooperative and interoperable program applications that offer information, which can be understood by the stakeholders. Additionally, rapidly accessible, predictive analytics provide feasible guidance when issues occur (Pan et al., 2017).
In principle, PIM consists of five fundamental stages, which include Project break down, Data management, PIM Big Data (PBD) architecture construction, Implementation, and PBD distribution. During the Project break down, each project stage is formulated as an individual data cluster, which can be deployed, assessed, processed and transferred independently. For example, the initial data clusters shall include design data, production data, procurement and tendering data, logistics data, rapid construction data and lifecycle management data. As a result, the data clusters are loosely coupled, thus providing the database information that can be categorised, classified and shared with the relevant party. The human data, physical data, project management data, facility data and cyber data will be analysed. The main goal of this stage is to differentiate and integrate the data based on the relevance of the information that is evaluated by the key stakeholders. This action can potentially enhance interdepartmental, cross-functional and cross-disciplinary data interaction; therefore, it adds value throughout the project.

During the next stage, PBD is further categorised into four main databases, which are the physical database, BIM database, Internet of Building Things (IoBT) database and maintenance management database. The physical database contains the information that is gathered through paperbased hardcopy documents, as well as the information not yet transferred into digital data. The BIM database covers the range of information from basic data to highly sophisticated implementations, which include 3D, 4D, 5D and 6D BIM applications.

The IoBT database comprises a range of smart data collected throughout the construction phases, which include geolocation tracking, monitoring of equipment, inventory, procurement management, quality inspection, real-time measuring and control and remote operation. The maintenance management database covers the information accumulated over repair, alteration, conversion, upgrading, scheduling, and budgeting of the lifecycle management activities (Calie, 2017).

The aforementioned data is collected and stored in the PIM data Processing Unit (PPU). PPU is not only for data acquisition, but also and most importantly it is a range of interoperable applications that actively processes data in real time and analyses huge amounts of data created from a variety of sources.

The main strategy of PPU is to process, integrate, transfer, share and store the real-time data. Also, it enhances collaboration and supports the decision-making activities 


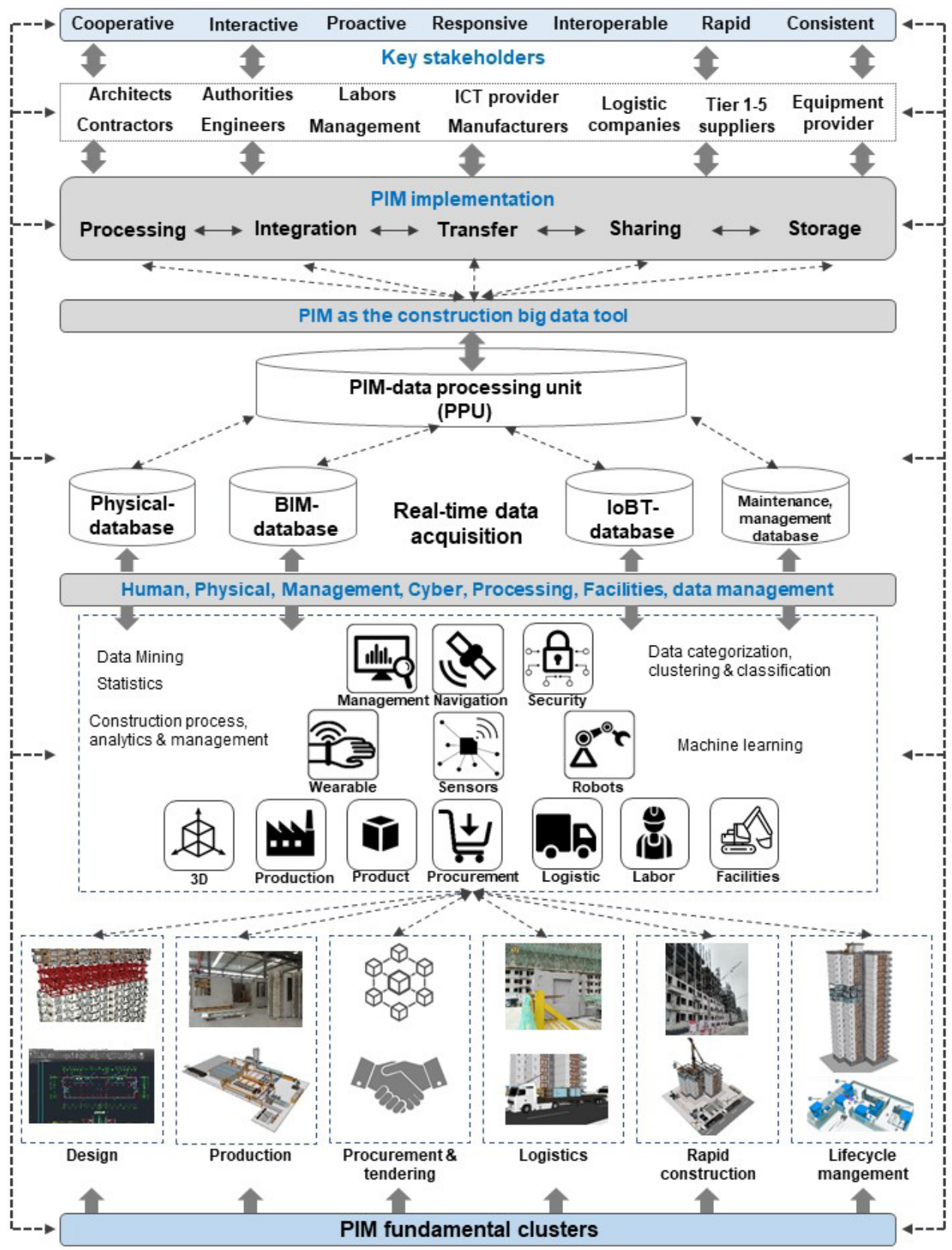

Fig. 1 Process information modelling concept.

by distributing the most relevant information to the right key stakeholders at the right time. At the time of this writing, PIM has been developed only as a conceptual model that demonstrates the overall concept, yet capable of offering basic instructions and data analysis that is based on the scenario created by the Hong Kong CIC project.

\section{Literature review}

The construction industry is dealing with significantly increasing data from various disciplines throughout the construction process. As mentioned earlier, the PIM proposal can be seen as a Big Data application. Utilisation of the applications can yield momentous benefits for an organisation or individuals undertaking a variety of complex construction projects. In this section, the literature review and theoretical background are briefly introduced.

\subsection{Big data}

In general, Big Data consists of two main activities, including Big Data Engineering (BDE) and Big Data Analytics (BDA). First, Big Data accumulates huge amounts of data and then processes them with tools such as Apache 
Hadoop (AH) and Apache Spark (AS). Secondly, Big Data storage is another crucial task, which decides whether the data is to be distributed or emerged.

Apache Hadoop: The AH is an open-source implementation of MapReduce (MR) (Dean and Ghemawat, 2008). It is designed for managing very large nodes of the database (White, 2012) on computer clusters that are constructed from commodity servers. Nowadays, many web-based brands are using an Apache Hadoop platform, such as Yahoo! (Vavilapalli et al., 2013) and Facebook (Borthakur et al., 2011).

Apache Spark: The AS is one of the many opensource cluster computing platforms for processing largescale data. It has gained increasing popularity recently due to the processing speed and user-friendly feature. It is a fault-tolerant and optimised by ApplicationProgramming-Interface (APIs) in interpreted high-level programming languages, such as Python, Java, R and Scala (Zaharia et al., 2016).

With the increasing amount of heterogeneous, autonomous parallel distributed sources and data, Big Data Analytics (BDA) has become crucial for many business disciplines. In general, BDA provides a new paradigm for handling, storage, management and access to those huge datasets (Miller et al., 2016).

Starfish: Commonly, starfish is a self-tuning system used by data scientists, business analysists and IT operators to visualise, optimise as well as strategise the $\mathrm{AH}$ application. It builds on the AH application and adapting users' needs to achieve better performance automatically (Herodotou et al., 2011).

\subsection{SODATO}

SODATO stands for Social Data Analytics Tool and was developed to provide a generic method to gather, store, process, analyse and summarise big social data, which accumulates through the organisation's social media platforms. It provides a strategic tool that actively interacts with the big social data (Hussain and Vatrapu, 2014).

\subsection{Program language}

During the development of the PIM concept, it was evident that there are various examples in computer software design, which have conceptual principles similar to those in PIM. A brief analysis is conducted to offer a reinterpretation of the PIM concept by using software programing concepts (Pan et al., 2017).
Service-oriented architecture (SOA) can be described as a loosely coupled program architecture designed specifically to meet the needs of an organisation (Arsanjani, 2004). By using communication protocols which provide services to another component and make connections between different software components over a network (Erl, 2004), the definition of a service can be viewed as a logical representation of a repeatable task. SOA is independent, self-contained, yet when combined with other software it forms the functionality of a large software application. The unit architecture in PIM also shares similarities with the service architecture in SOA (Patrick et al., 2005). It is beneficial when linking an agent that is influenced by the unit data or service, and the changes would influence the individual agent's capabilities or responsibilities (Pan et al., 2017).

The Microservice architecture is a programming concept inspired by SOA. Instead of a traditional monolith software application, Microservices provides groups of independent program components that are operated and deployed separately, yet are based on precise protocols and dedicated memories. The Microservice architecture has gain popularity in the recent years; it has potential to contribute to the development of the PIM concept. However, there is limited research that has been conducted that emphasised the topic (Nwana, 1996). Therefore, further validation through application use-case is necessary (Dragoni et al., 2017).

Service discovery or service discovery protocols (SDP) is an emerging field in the area of ubiquitous computing (Richard and Spencer, 2001). They provide a mechanism, which allows automatic detection of service offered by any node in the network. In other words, service discovery is the action of finding a service provider for a requested service (Czerwinski et al., 1999). Service discovery can potentially operate as a search engine for the PIM architecture (Pan et al., 2017).

\subsection{System integration}

Due to the complexity of the construction projects, the multidisciplinary stakeholders and implementation of heterogeneous data through the use of different software and hardware are hard to manage. Data integration becomes very critical, which enables smooth operation and effective collaboration (Shen et al., 2010). A number of challenges regarding the data integration within the construction, can be summarised as below (Eadie et al., 2013):

- There is a lack of transferability and interoperability between data, systems, programs and methodologies. 
- The comprehensive universal management tool for different phases of the construction project is still not available.

- Lifecycle management issues are often not emphasised. Operation, maintenance, dismantling and recycling are taken in consideration less.

- There is no common tool to manage health safety measures, and predictions of operation hazard are not available.

As a future construction IT system, the PIM application will ensure construction information is available on demand and to can be transferred to the desired stakeholders at the right time. All project partners, construction tools, equipment and machinery will be interconnected through integrated management systems. This will enhance planning, enable rapid response and optimise overall running of the project (Rezgui and Zarli, 2006).

\section{5 $\operatorname{Actyx}^{\circledR}$}

Acty $x^{\circledR}$ is a company based in Munich, which offers extensive ranges of software applications aimed to increase productivity, seamless integration, reduce lead-time, and identify bottlenecks in various industries. The research team noticed there are some applications and products the company offers that seem to share the same objectives as PIM. The relevant applications will be described briefly in the following section. Actyx ${ }^{\circledR}$ Manual Work Logging is an operation that automatically generates real-time operational data based on the manual activities on the factory shop floor. This feature is similar to the real-time construction data acquisition offered in the PIM concept. Actyx ${ }^{\circledR}$ Manual Work Logging is designed to use sensors to gather real-time machine operation data.

The application also offers early error detection and prevention. It shares the similar quality as PIM production data categorization, clustering and construction process analytics. Acty $x^{\circledR}$ Material Movement Logging is an application that offers a transparent, accurate and practical logging of the material logistic flow in the warehouse. This function is similar to logistic tracking and documentation in PIM.

The aforementioned applications demonstrate similar objects as the proposed PIM concept, however, they are not available for the construction industry. In order to implement them, the application needs to be customised, which might be a costly task (Actyx, 2018).

\section{6 АTHMOS}

A novel technique in machine learning and modular data analysis framework, which is called Automated Telemetry Health Monitoring System (ATHMoS) for space operation, was developed by the Data Mining team at the German Space Operation Center (GSOC). The principle of ATHMos is to train the system to learn the correct historic data or on-going mission lifetime data, and then to automatically detect and analyse new telemetry data as well as identify anomalies. If any anomalous behaviour is detected, the ground based engineers can be notified in advance so that they can organise and decide what actions to take. ATHMoS was an initial study that demonstrates the potential of using neural networks and machine learning in satellite telemetry monitoring. The space operation system relies on extremely accurate data detection and transformation, in contrast, the construction sector is somewhat tolerable. Nevertheless, some machine learning algorithms in other industries are being replaced or augmented with deep learning due to the technological development in the software engineering sector. The big data and machine learning have only been adopted in the space operation sector (O'Meara et al., 2018). The authors consider this as an inspiration for future PIM development and use of Artificial Intelligence in the construction industry.

\section{PIM concept development for external wall painting task}

The Technical University of Munich (TUM) is commissioned by the Construction Industry Council of Hong Kong (CIC) to research and develop construction robots and automation strategies that are tailor-made for the public housing construction project in Hong Kong. An extensive case study was conducted at Ngan Kwong Wan Road in Mui Wo, Hong Kong that was erected by Hip Hing Engineering Co., Ltd. The project consists of one 14-storey and a 16-storey residential building. In this paper, the project team uses the 16-storey building as a case study to investigate how to implement PIM application to carry out the external façade painting task by using a painting robot, which will be developed during the CIC project.

The identified key stakeholders, who are involved in the exterior painting task include:

1. Contractor (C)

2. Project manager (PM)

3. On-site worker (W)

4. Equipment supplier (ES) 
5. Robot supplier (RS)

6. Painting robot (PR)

7. Paint / material supplier (PS)

8. Logistics company (L)

9. Inspector (I).

Each stakeholder has a particular interest in the project as well as a specific task. PIM aims to collect the most relevant data and to process and distribute it among the most relevant stakeholders. The following section will demonstrates this process in detail (Fig. 2).

First, the detailed breakdown of the working sequences, involved stakeholders, and data for the exterior wall painting task are analysed.

The main purpose of the proposed approach is to provide an automated process through accurate information flow from the design phase to the construction phase. Extension of the data stored in the BIM software is achieved by developing property sets (Fig. 3).

Pset_CIC_Painting is generated for schema IfcArchitectureDomain in the domain layer of the general IFC architecture. It has the properties of PaintingRobot, PaintingMaterial and MaxHeight. Data types for the criteria are set as IfcBoolean, IfcPropertyEnumeratedValue and IfcReal, respectively. PaintingRobot finds out whether the façade painting is performed by a robot or not, MaxHeight refers to the maximum height of the external wall and PaintingMaterial includes the paint information.

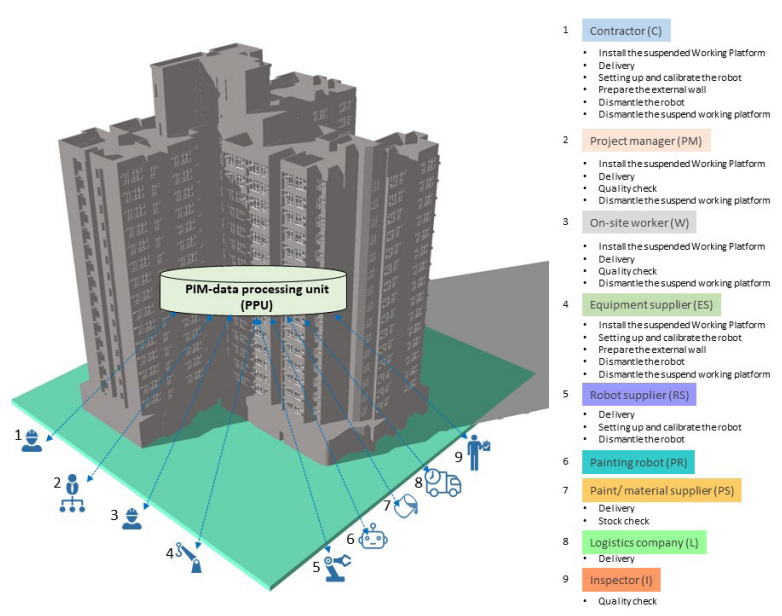

Fig. 2 Stakeholders and their dedicated tasks

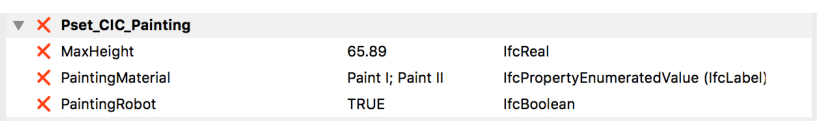

Fig. 3 Property sets
The CIC template file (Fig. 4) including the extended properties and building materials / composites for façade painting is created.

The user should assign each property using the IFC Manager menu. Pset_CIC_Painting is applicable to the project entity. Then, the BIM file is exported as an IFC format file and transferred to an Excel file through IFC File Analyzer (IFA) (Lipman, 2017) (Fig. 5). In case of PaintingRobot being TRUE, the relevant data for the corresponding process can be extracted for the painting with automatic application.

Fig. 6 demonstrates the workflow for façade painting task carried out by a painting robot.

Table 1 presents the corresponding data for each task description.

\section{Conclusion}

This paper provides an overview of the PIM concept and introduces a use case for how PIM applications would be operated while using the construction robotics on-site. The conventional BIM applications are designed to collect and distribute basic information about the construction project. However, they are not adequate in dealing with the situation if the construction robots are implemented.

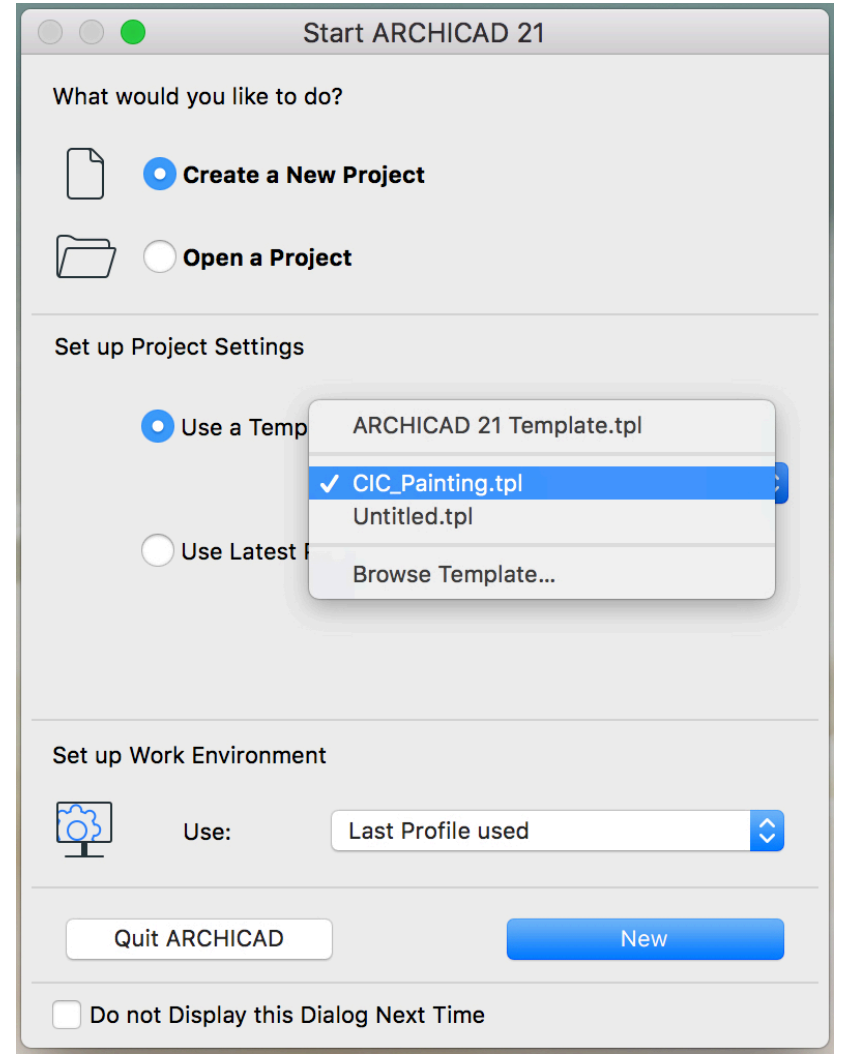

Fig. 4 CIC template file 

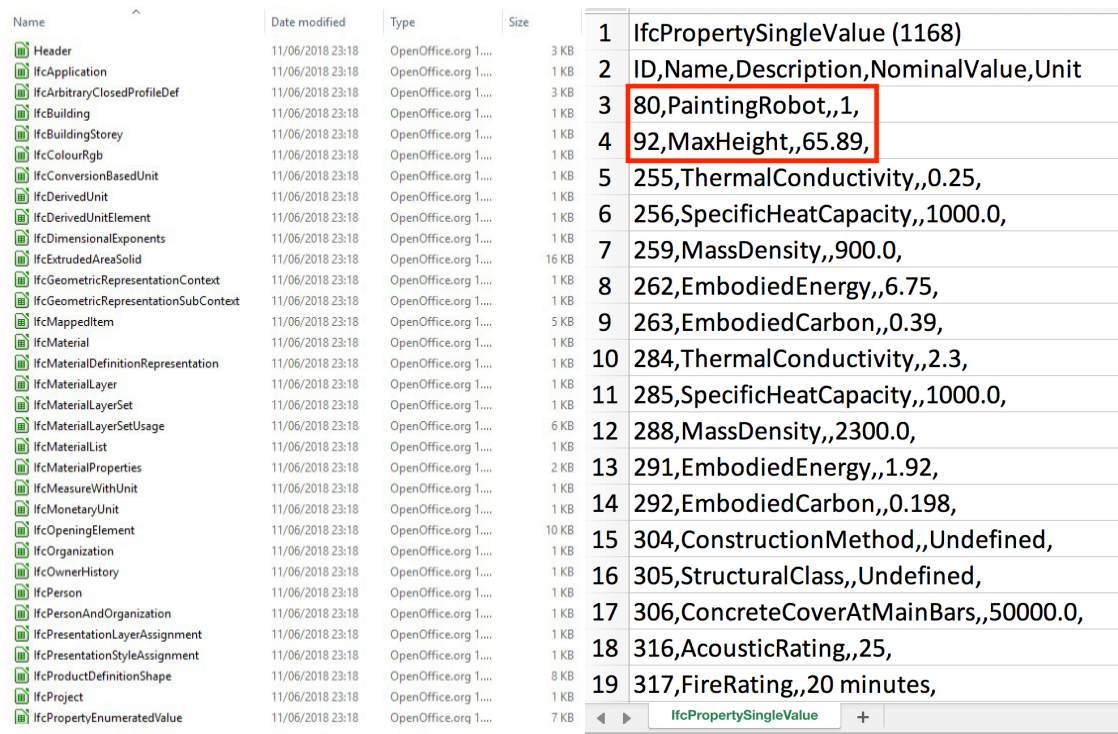

Fig. 5 Transferred IFC files into spreadsheets

Since they have a limited function in understanding the operational data generated by sensors and robots, there is a need of a more comprehensive approach. The concept of PIM enhances all aspects of the construction operations

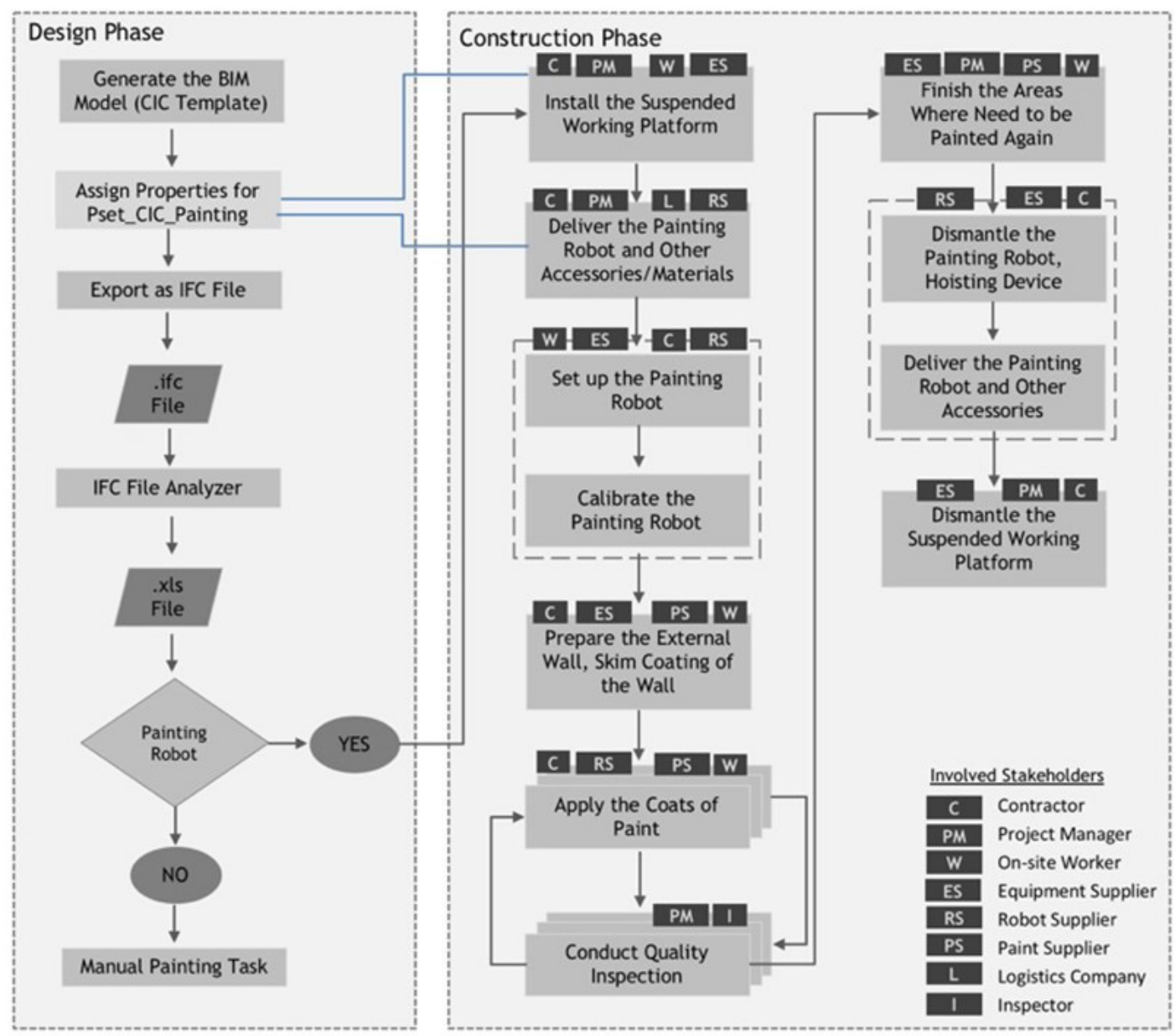

Fig. 6 Flowchart for façade painting 
Table 1 Robot-oriented Painting Task and Related Data

\begin{tabular}{|c|c|}
\hline Task description & Data bank \\
\hline $\begin{array}{l}\text { Installing the suspended } \\
\text { working platform }\end{array}$ & $\begin{array}{l}\text { Product, logistic, planning, bidding, } \\
\text { labour, equipment, health \& safety, } \\
\text { repair \& maintenance data }\end{array}$ \\
\hline $\begin{array}{l}\text { Delivering the painting } \\
\text { robot and other accessories }\end{array}$ & $\begin{array}{l}\text { Robot, logistic, planning, bidding, } \\
\text { navigation, distribution data }\end{array}$ \\
\hline $\begin{array}{l}\text { Setting up \& calibrating } \\
\text { the painting robot }\end{array}$ & $\begin{array}{l}\text { Robot, planning, equipment, repair \& } \\
\text { maintenance }\end{array}$ \\
\hline $\begin{array}{l}\text { Cleaning and preparing the } \\
\text { external wall, skim coating } \\
\text { of the wall }\end{array}$ & $\begin{array}{l}\text { Robot, bidding, planning, equipment, } \\
\text { repair \& maintenance, labour }\end{array}$ \\
\hline $\begin{array}{l}\text { Applying the first coat of } \\
\text { paint }\end{array}$ & $\begin{array}{l}\text { Robot, paint supply, planning, } \\
\text { equipment, repair \& maintenance, } \\
\text { labour }\end{array}$ \\
\hline $\begin{array}{l}\text { Conducting quality } \\
\text { inspection }\end{array}$ & Robot, planning, equipment, authority \\
\hline $\begin{array}{l}\text { Applying the second coat } \\
\text { of paint }\end{array}$ & $\begin{array}{l}\text { Robot, paint supply, planning, } \\
\text { equipment, repair \& maintenance, } \\
\text { labour }\end{array}$ \\
\hline $\begin{array}{l}\text { Conducting quality } \\
\text { inspection }\end{array}$ & Robot, planning, equipment, authority \\
\hline $\begin{array}{l}\text { Applying the final coat of } \\
\text { paint }\end{array}$ & $\begin{array}{l}\text { Robot, paint supply, planning, } \\
\text { equipment, repair \& maintenance, } \\
\text { labour }\end{array}$ \\
\hline $\begin{array}{l}\text { Conducting quality } \\
\text { inspection }\end{array}$ & Robot, planning, equipment, authority \\
\hline $\begin{array}{l}\text { Finishing the areas where } \\
\text { need to be painted again }\end{array}$ & $\begin{array}{l}\text { Robot, paint, planning, equipment, } \\
\text { repair \& maintenance, labour }\end{array}$ \\
\hline $\begin{array}{l}\text { Dismantling the painting } \\
\text { robot, hoisting device }\end{array}$ & $\begin{array}{l}\text { Robot, planning, equipment, repair \& } \\
\text { maintenance }\end{array}$ \\
\hline $\begin{array}{l}\text { Dismantling the suspended } \\
\text { working platform }\end{array}$ & $\begin{array}{l}\text { Product, logistic, planning, labour, } \\
\text { equipment, health \& safety, repair \& } \\
\text { maintenance data }\end{array}$ \\
\hline
\end{tabular}

by not only collecting and distributing the data, but also proceeding and analysing them for optimising the decision-making during each task. The proposed approach provides systematic information flow and management for the construction phase of the project life cycle via incorporating the BIM data.

This design and construction data integrated solution enables improved construction process management. In the current situation, BIM facilitates the design stage

\section{References}

Actyx "Actyx", [online] Available at: https:/www.actyx.io/en/ [Accessed: 28th May 2018]

Arsanjani, A. (2004) "Service-oriented modeling and architecture", IBM Developer Works, [online] Available at: https://www.ibm.com/ developerworks/library/ws-soa-design1/index.html [Accessed: 15th April 2018]

Azhar, S., Nadeem, A., Mok, J. Y. N., Leung, B. H. Y. (2008) "Building Information Modeling (BIM): A New Paradigm for Visual Interactive Modeling and Simulation for Construction Projects", In: First International Conference on Construction in Developing Countries, Karachi, Pakistan, pp. 435-446. of a construction project and provides accurate project information, and continuation of the construction processes is separately handled based on the design data. Nevertheless, since most of the project delays and consequently cost overruns arise from the construction phase, more integrated and automated methodologies should be adopted. For instance, if the correct material / equipment information is gathered and transferred to the corresponding stakeholder as rapid as possible, the necessary actions can be made earlier than the actual construction to prevent any possible delays (e.g. in the façade painting case, checking the supplier of the paint automatically and in case of unavailability of that supplier, searching the possible ones via warning the related parties).

Moreover, the paper functions as an eye-opener to the construction industry through a demonstration on how to carry out BDE and BDA activities that associate with the vast amounts of heterogeneous data.

As mentioned earlier, at the time of this writing, PIM is limited to the conceptualisation. PIM is only developed as a conceptual idea that establishes the overall concept, yet will not bear on its ability of offering basic documentation tasks or providing instructions and data analysis by using existing Microsoft Excel tool. In addition, a comprehensive research project is needed to develop the concept further. It is important to use a real case study to develop the hardware and software environment, which will be required by PIM developers. Due to the given time and resources, the potential of the PIM application as a future Big Data application for the future construction industry, and privacy or data protection issues have not been discussed in detail.

\section{Acknowledgement}

The authors would like to sincerely thank the Construction Industry Council of Hong Kong (CIC) and Hip Hing Engineering Co., Ltd for providing valuable information that supported this research. The research was also funded by TUBITAK.

Borthakur, D., Gray, J., Sarma, J. S., Muthukkaruppan, K., Spiegelberg, N., Kuang, H., Ranganathan, K., Molkov, D., Menon, A., Rash, S., Schmidt, R., Aiyer, A. (2011) "Apache Hadoop goes realtime at Facebook", In: ACM SIGMOD International Conference on Management of Data, Athens, Greece, pp. 1071-1080. https://doi.org/10.1145/1989323.1989438

Calie, P. (2017) "Innovation Insight: The Impact of Emerging Technologies on the Construction Industry", [pdf] DeltaHedron, Available at: https://www.deltahedron.co.uk/wp-content/ uploads/2017/08/DeltaHedron-Innovation-Insight_Impact-ofemerging-technologies-on-construction_No-4-17_Aug-2017.pdf [Accessed: 10th January 2018] 
Czerwinski, S. E., Zhao, B. Y., Hodes, T. D., Joseph, A. D., Katz, R. H. (1999) "An architecture for a secure service discovery service", In: $5^{\text {th }}$ Annual ACM/IEEE International Conference on Mobile Computing and Networking, Seattle, WA, USA, pp. 24-35. https://doi.org/10.1145/313451.313462

Dean, J., Ghemawat, S. (2008) "MapReduce: simplified data processing on large clusters", Communications of the ACM, 51(1), pp. 107-113. https://doi.org/10.1145/1327452.1327492

Dossick, C. S., Neff, G. (2010) "Organizational Divisions in BIMEnabled Commercial Construction", Journal of Construction Engineering and Management, 136(4), pp. 459-467. https://doi.org/10.1061/(ASCE)CO.1943-7862.0000109

Dragoni, N., Giallorenzo, S., Lafuente, A. L., Mazzara, M., Montesi, F., Mustafin, R., Safina, L. (2017) "Microservices: Yesterday, Today, and Tomorrow", In: Mazzara, M., Meyer, B. (eds.) Present and Ulterior Software Engineering, Springer, Cham, Switzerland, pp. 195-216.

https://doi.org/10.1007/978-3-319-67425-4_12

Eadie, R., Browne, M., Odeyinka, H., McKeown, C., McNiff, S., (2013) "BIM implementation throughout the UK construction project lifecycle: An analysis", Automation in Construction, 36, pp. 145-151. https://doi.org/10.1016/j.autcon.2013.09.001

Eastman, C. M., Teicholz, P., Sacks, R., Liston, K. (2013) "BIM Handbook: A Guide to Building Information Modeling for Owners, Managers, Architects, Engineers, Contractors and Fabricators", 2nd ed., John Wiley and Sons, Hoboken, NJ, USA.

Elkaseer, A., Salama, M., Ali, H., Scholz, S. (2018) "Approaches to a Practical Implementation of Industry 4.0", In: ACHI 2018: The Eleventh International Conference on Advances in ComputerHuman Interactions, Rome, Italy, pp. 141-146.

Erl, T. (2004) "Service-Oriented Architecture: A Field Guide to Integrating XML and Web Services", [pdf] Prentice Hall PTR, Available at: http://disi.unal.edu.co/dacursci/sistemasycomputacion/docs/SWEBOK/SOA_Service-Oriented $\% 20$ Architecture $\% 20-\% 20$ Field $\% 20$ Guide $\% 20$ To\%20Integrating $\% 20$ Xm1\%20And\%20Web\%20Services_2004_Prentice.pdf [Accessed: 25th March 2018]

Harty, C., Throssell, D., Jeffrey, H., Stagg, M. (2010) "Implementing building information modeling: a case study of the Barts and the London hospitals", In: The International Conference on Computing in Civil and Building Engineering, Nottingham, UK, Electronic Proceedings. [online] Available at: http://www.engineering.nottingham.ac.uk/icccbe/proceedings/pdf/pf93.pdf [Accessed: 27th December 2017]

Herodotou, H., Lim, H., Luo, G., Borisov, N., Dong, L., Cetin, F. B., Babu, S. (2011) "Starfish: a Self-tuning System for Big Data Analytics", In: Conference on Innovative Data Systems Research (CIDR) 2011, Asilomar, California, USA, pp. 261-272. [online] Available at: http://cidrdb.org/cidr2011/Papers/CIDR11_Paper36. pdf [Accessed: 13th January 2018]

Higgin, G., Jessop, N. (eds.) (2013) "Communications in the Building Industry: The Report of a Pilot Study", 1st ed., Routledge, Abingdon, UK.
Hussain, A., Vatrapu, R. (2014) "Social Data Analytics Tool (SODATO)", In: Tremblay, M. C., VanderMeer, D., Rothenberger, M., Gupta, A., Yoon, V. (eds.) Advancing the Impact of Design Science: Moving from Theory to Practice, DESRIST 2014, Lecture Notes in Computer Science Book Series, Springer, Cham, Switzerland, pp. 368-372. https://doi.org/10.1007/978-3-319-06701-8_27

Jernigan, F. E. (2008) "Big BIM, Little Bim: The Practical Approach to Building Information Modeling: Integrated Practice Done the Right Way!", 1st ed., 4Site Press, Salisbury, Maryland, USA.

Lipman, R. (2017) "IFC File Analyzer Software", Journal of Research of the National Institute of Standards and Technology, 122(15), pp. 1-2. https://doi.org/10.6028/jres.122.015

Miller, J. A., Bowman, C., Harish, V. G., Quinn, S. (2016) "Open Source Big Data Analytics Frameworks Written in Scala", In: IEEE International Congress on Big Data (BigData Congress), San Francisco, USA, pp. 389-393

https://doi.org/10.1109/BigDataCongress.2016.61

Nwana, H. S. (1996) "Software agents: An overview", The Knowledge Engineering Review, 11(3), pp. 205-244. https://doi.org/10.1017/S026988890000789X

O'Meara, C., Schlag, L., Wickler, M. (2018) "Applications of Deep Learning Neural Networks to Satellite Telemetry Monitoring", In: 2018 SpaceOps Conference, The $15^{\text {th }}$ International Conference on Space Operations, Marseille, France, pp. 1-16. https://doi.org/10.2514/6.2018-2558

Pan, W., Ilhan, B., Bock, T. (2018) "Process Information Modelling (PIM) for Public Housing Construction Project in Hong Kong", In: Creative Construction Conference 2018, Ljubljana, Slovenia, pp. 74-81. https://doi.org/10.3311/CCC2018-011

Pan, W., Langosch, K., Bock, T. (2017) "Development of the Process Information Modelling in the Construction Project: A Case Study of the ZERO-PLUS Project", In: 34 $4^{\text {th }}$ International Symposium on Automation and Robotics in Construction (ISARC 2017), Taipei, Taiwan, pp. 775-782. https://doi.org/10.22260/ISARC2017/0108

Patrick, P. B., Aletty, A., Kasi, J., Kapoor, C., Urhan, T., Mihic, M., BEA Systems, Inc. (2005) "Service Oriented Architecture", San Jose, CA, USA, U.S. Patent Application Ser. No. 11/133,022.

Rezgui, Y., Zarli, A. (2006) "Paving the Way to the Vision of Digital Construction: A Strategic Roadmap", Journal of Construction Engineering and Management, 132(7), pp. 767-776. https://doi.org/10.1061/(ASCE)0733-9364(2006)132:7(767)

Richard, G. G., Spencer, M. (2001) "Service Discovery Protocols and Programming", 1st ed., McGraw-Hill Professional, New York, NY, USA.

Shen, W., Hao, Q., Mak, H., Neelamkavil, J., Xie, H., Dickinson, J., Thomas, R., Pardasani, A., Xue, H. (2010) "Systems integration and collaboration in architecture, engineering, construction and facilities management: A review", Advanced Engineering Informatics, 24(2), pp. 196-207. https://doi.org/10.1016/j.aei.2009.09.001 
Trist, E. L., Bamforth, K. W. (1951) "Some Social and Psychological Consequences of the Longwall Method of Coal-Getting: An Examination of the Psychological Situation and Defences of a Work Group in Relation to the Social Structure and Technological Content of the Work System", Human Relations, 4(1), pp. 3-38. https://doi.org/10.1177/001872675100400101

Vavilapalli, V. K., Murthy, A. C., Douglas, C., Agarwal, S., Konar, M., Evans, R., Graves, T., Lowe, J., Shah, H., Seth, S., Saha, B., Curino, C., O'Malley, O., Radia, S., Reed, B., Baldeschwieler, E. (2013) "Apache Hadoop YARN: Yet another resource negotiator", In: $4^{\text {th }}$ Annual Symposium on Cloud Computing, Santa Clara, CA, USA, ACM 978-1-4503-2428-1.

https://doi.org/10.1145/2523616.2523633
White, T. (2012) "Hadoop: The Definitive Guide", [pdf] 3rd ed., O'Reilly Media, Inc., Sebastopol, CA, USA. Available at: http:// javaarm.com/file/apache/Hadoop/books/Hadoop-The.Definitive. Guide_4.edition_a_Tom.White_April-2015.pdf [Accessed: 4th February 2018]

Zaharia, M., Xin, R. S., Wendell, P., Das, T., Armbrust, M., Dave, A., Meng, X., Rosen, J., Venkataraman, S., Franklin, M. J., Ghodsi, A., Gonzalez, J., Shenker, S., Stoica, I. (2016) "Apache Spark: a unified engine for big data processing", Communications of the ACM, 59(11), pp. 56-65.

https://doi.org/10.1145/2934664 